The word 'comprehensive' in the title thus represents no idle boast.

Insofar as this single volume covers the entire curriculum recommended by his official examining boards, it will no doubt serve a useful purpose for the American postgraduate student. Its value for his British counterpart is more debatable. From this point of view, the relevance of much of the text is limited by its heavy reliance on a purely national frame of reference. To quote again from the preface, 'The organising principles ... are derived from certain distinguishing characteristics of American life that are rooted in our history and from the guidelines for the development of our social institutions': to wit, pluralism, pragmatism and progressivism. One consequence of this socio-cultural background is to be found in the preoccupation with psycho-analytic doctrine which runs through the book, belying the editors' professed eclecticism. Another is the curiously Nineteenth Century attitude to social matters, culminating in a chapter entitled 'Treatment of the Impoverished'. 'It is now felt', we read, 'that the poor deserve many things they do not have or do not seem to be able to get through their own efforts. Among these things are treatment for mental illness'. Quite so, and in particular attention to the social problems of chronic schizophrenia, senile psychosis and mental subnormality, all here relatively neglected.

Apart from this lack of balance, the text is of uneven standard. While some individual contributions are excellent, others are superficial in content and diffuse in style. A single sample, chosen at random, will suffice to give the flavour. In the management of Anorexia nervosa, we are told, the malnutrition can usually be ignored 'unless the weight loss is excessive', while treatment of the mental state 'must be highly individualised, ranging from somatic modalities to psycho-analysis, depending on the case'.

It seems doubtful if this kind of omnium gatherum can ever be wholly successful. What the postgraduate student needs is a shorter, more compact text, a sound bibliography which provides references to all the important original work, and access to a good library.

\section{Neurophysiology of Postural Mechanisms}

By Tristan D. M. Roberts. Pp. xvii + 354, illustrated. London: Butterworths, 1967. $£ 5$.

\section{The Basal Ganglia and Posture}

By James Purdon Martin. Pp. xiii + 152, illustrated. London: Pitman Medical, 1967. 80s.

These two publications offer an interesting comparison as to the way in which the problem of the control of posture can be approached.

The work by Roberts is concerned with the normal physiology of posture with particular reference to the cat. It provides a very able treatment of the recent substantial advances in our knowledge as to the morphology of the muscle spindle and the activity of the spindle system as an example of a servo mechanism. He begins with a general outline of certain basic neurophysiological principles, these chapters having also been published separately. He then goes on to consider the the mammalian muscle spindle, the stretch reflex and the muscle servo, and this is followed by a fascinating chapter on general problems of standing and locomotion. Consideration of the sense organs of the labyrinth precedes the final chapter on central coordinating mechanisms.

A completely different approach is provided by Purdon Martin's book which consists of a clinical and clinico-pathological analysis of patients with extrapyramidal disorders in which the results of the type of electrophysiological analysis presented by Roberts are all but ignored. It seems likely that the study of the breakdown of postural control in patients with neurological disorders will be a rewarding source of information as to the way in which posture is normally controlled in man, but it is clear that much remains to be achieved in this direction. Nevertheless, from the purely clinical standpoint, there is much of interest in Purdon Martin's carefully documented cases.

\section{The Electrical Activity of the Nervous System}

By Mary A. B. Brazier. Third Edition. Pp. xiv +317 , illustrated. London: Pitman Medical, 1968. 45s.

Since the second edition of Dr Brazier's textbook was published in 1960, there have been considerable advances in this field and the work has accordingly been substantially and successfully revised. Dr Brazier has a very readable style and presents often difficult material with an easy clarity.

The early chapters consist of a consideration of the electrical activity of single axons and aggregates of axons and then progresses to the phenomena of transmission at synapses and at the neuromuscular junction. This is followed by a discussion of the electrical activity of the spinal cord and brain and the electrical aspects of sensory activity. The final chapters deal with the normal and abnormal electroencephalogram in man. It is perhaps somewhat arbitrary to consider only the abnormal EEG and not other pathological derangements of the electrical activity of the nervous system. However, since the EEG is Dr Brazier's particular field of interest, this is understandable.

The reviewer has no doubt that this further edition will continue to uphold the fully deserved reputation of the book, which has already firmly established itself as a classic.

\section{Handbook of Diagnostic Cytology}

By Helena E. Hughes and T. C. Dodds. Pp. 225 , illustrated. Edinburgh and London: E. \& S. Livingstone. 1968. $£ 5$.

The success of diagnostic cytology has led to its rapid development. Expansion of services is often limited to the speed of training junior technicians and doctors. A problem under research one year can become part of a routine investigation the next. The many sites of cells available for examination mean that clinicians other than gynaecologists are demanding a cytology service. This book is a valuable guide for trainees in cytology. It is also commended to any doctor who wants to appreciate the scope and limitations of cytology, for eventually the clinician will be responsible for collection of the specimens.

One-quarter of the book is gynaecological cytology. One-half covers cytology of the respiratory, gastro-intestinal, and urinary tracts, the breast and the body fluids. The remainder is devoted to comment on radiation effect and the organisation of a cytology service. The text is didactic. There is a bibliography but no specific cross-references. Anatomical, pathological, and research details are an omission admitted by the authors. The requirements in these fields will vary widely according to the type of reader and their inclusion would change the character of the book making it a reduplication of the standard cytology textbooks.

Photomicrographs are the major part of any book on cytology. In this case they are all in colour which at times is rather harsh, but, as illustration of diagnostic detail, the photographs are most satisfactory. The publishers are to be commended for not isolating picture from its legend and yet retaining paragraphs intact. Photographs and text can be 'read' smoothly.

This book is more suitable for the student than for the established cyto-technician or cyto-pathologist; it covers an enormous subject concisely. It is remarkably cheap for such a high quality book. 